\title{
Vitamin $E$ as an IgE inhibitor: stability during cold storage of human milk
}

\author{
G. García-Llatas ${ }^{1}$, R. Lacomba ${ }^{1}$, F. Ortega ${ }^{1}$, A. Alegría ${ }^{1}$, R. Barberá ${ }^{1}$ and D. Silvestre ${ }^{2}$ \\ ${ }^{1}$ Nutrition and Food Chemistry, University of Valencia, Avda. Vicente Andrés Estellés s/n, 46100 Burjassot, Valencia, Spain \\ and ${ }^{2}$ Food Science, Cardenal Herrera-CEU University, Avda. Seminario s/n, 46113 Moncada, Valencia, Spain
}

Human breast milk is recommended as the unique food for neonates based on its known properties. When the production of milk by the mother is not sufficient or the mother is not able to feed her child for professional reasons, milk banks or the mothers' practice of collecting their own milk are the existing alternatives for breast-feeding. In both situations cold storage (refrigeration or freezing) can be used in neonatal units, at home and in human milk banks ${ }^{(1,2)}$.

Many micronutrients with immunological properties are found in breast milk. In addition to its antioxidant activity, vitamin E has been shown to be associated with a decrease in the frequency of allergen sensitisation by the inhibition of IgE responses to allergic stimuli; asthma, rhinitis and hayfever are atopic disorders characterised by raised serum IgE and skin sensitisation to common environmental allergens $^{(3)}$.

The stability of vitamin E contents can be influenced by temperature and time of storage. Thus, the aim of the present study was the evaluation of vitamin E contents during cold storage of human milk. Fourteen samples of mature human milk from healthy and wellnourished women were collected from a breast-feeding workshop. Each sample was divided in three aliquots, one of which was analysed just after collection (F) and the others were stored under the conditions used at home (refrigerating at $2-4^{\circ} \mathrm{C}$ for $48 \mathrm{~h}$; R) and at milk banks (freezing at $-18^{\circ} \mathrm{C}$ for $15 \mathrm{~d} ; \mathrm{Fz}$ ).

$\alpha-, \beta-, \gamma$ - and $\delta$-Tocopherols were quantified simultaneously in the milk samples by a normal-phase HPLC method with fluorescence detection $^{(4)}$ (four determinations per sample). The results (ranges) are shown in the Table.

\begin{tabular}{lccc}
\hline $\begin{array}{l}\text { Tocopherols } \\
(\mu \mathrm{g} / 100 \mathrm{ml} \text { human milk })\end{array}$ & $\mathrm{F}$ & $\mathrm{R}$ & $\mathrm{Fz}$ \\
\hline$\alpha$ & $209.9-264.0$ & $169.3-223.4$ & $193.2-247.3$ \\
$\beta$ & $34.8-57.5$ & $44.1-68.1$ & $25.0-47.7$ \\
$\gamma$ & $54.2-66.0$ & $49.6-61.4$ & $55.9-67.8$ \\
$\delta$ & $9.25-18.0$ & $14.1-22.8$ & $9.43-18.2$ \\
\hline
\end{tabular}

One-way (storage temperature) ANOVA applied to the results showed no significant differences $(P<0.05)$ between the treatments studied. Thus, the inhibition of IgE responses provided by vitamin E from human milk is maintained after it is refrigerated or stored frozen.

This study is part of the project PRUCH 03/36 (2003-2004) supported financially by Cardenal Herrera-CEU University.

1. Human Milk Banking Association of North America (2006) Best Practice for Expressing, Storing and Handling Human Milk in Hospitals, Homes and Child Care Settings, 2nd ed. [F Jones and MR Tully, editors]. Raleigh, NC: HMBANA.

2. Lindemann PC, Foshaugen I \& Lindemann R (2004) Arch Dis Child Fetal Neonatal Ed 89, F440-F441.

3. Fogarty A, Lewis DS, Weiss S \& Britton J (2000) Lancet 356, 1573-1574.

4. Rodrigo N, Alegría A, Barberá R \& Farré R (2002) J Chromatogr 947A, 97-102. 